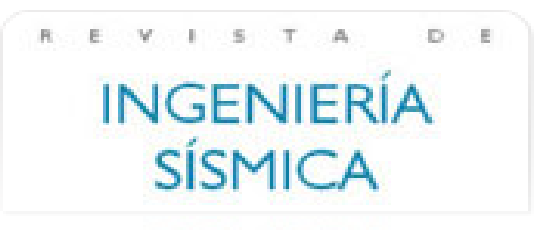

Revista de Ingeniería Sísmica

ISSN: 0185-092X

javiles@tlaloc.imta.mx

Sociedad Mexicana de Ingeniería Sísmica

México

Escobar Sánchez, José Alberto; Magno Cisneros, Carlo; Gómez Martínez, Roberto Evaluación de criterios de diseño por torsión sísmica estática para edificos de varios pisos

Revista de Ingeniería Sísmica, núm. 71, julio-diciembre, 2004, pp. 63-89

Sociedad Mexicana de Ingeniería Sísmica

Distrito Federal, México

Disponible en: http://www.redalyc.org/articulo.oa?id=61807103

Cómo citar el artículo

Número completo

- Más información del artículo

- Página de la revista en redalyc.org

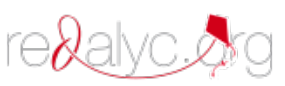

Sistema de Información Científica

Red de Revistas Científicas de América Latina, el Caribe, España y Portugal Proyecto académico sin fines de lucro, desarrollado bajo la iniciativa de acceso abierto 


\title{
EVALUACIÓN DE CRITERIOS DE DISEÑO POR TORSIÓN SÍSMICA ESTÁTICA PARA EDIFICOS DE VARIOS PISOS
}

\author{
José Alberto Escobar Sánchez, Carlo Magno Cisneros y Roberto Gómez Martínez ${ }^{(1)}$
}

\begin{abstract}
RESUMEN
Se presenta una evaluación de diferentes propuestas para reducir la cantidad de cálculos en el análisis por computadora de modelos tridimensionales de edificios por torsión sísmica estática. Fundamentalmente, se busca reducir lo laborioso del proceso del análisis realizado con programas de cómputo al incluir la excentricidad accidental. Se describen las bases teóricas que dan origen al análisis por torsión sísmica estática de estructuras de edificios, se estudian propuestas recientes enfocadas a realizar este análisis mediante el uso de programas comerciales de análisis estructural, y se propone y evalúa un nuevo procedimiento que, sin perder precisión en los cálculos, simplifica notablemente este tipo de análisis. Se presentan ejemplos en los que se aplican las diferentes propuestas hechas y se discuten sus ventajas y desventajas.
\end{abstract}

\section{SUMMARY}

An evaluation of different proposals to reduce the number of calculations in the analysis of threedimensional models of buildings for static seismic torsion is presented. Fundamentally what the proposals look for is to reduce the laborious of the process of analysis carry out with computer programs when the accidental eccentricity concept is included. In the present work, the theoretical basis of the analysis for static seismic torsion of structures of buildings are described, and the most recent proposals focused to carry out this analysis by means of the use of commercial programs of structural analysis are studied. A new procedure is proposed an evaluated that simplifies this analysis type notably without losing precision in the calculations. Finally, the different proposals are applied to two examples and the advantages and disadvantages on their use are discussed.

\section{INTRODUCCIÓN}

A pesar de que existen recomendaciones explícitas al respecto, el análisis de estructuras sometidas a torsión sísmica es un aspecto que algunos ingenieros pueden manejar intuitivamente (Damy, 1989), con todas las desventajas que esta práctica puede implicar. Para evitar lo anterior, se han propuesto métodos que simplifican este tipo de análisis. Debido a sus características, el análisis por torsión de estructuras se simplificó de ser un problema dinámico a uno estático. Aún más, debido a lo laborioso del análisis estructural de un modelo tridimensional de una estructura,

Artículo recibido el 7 de septiembre de 2003 y aprobado para su publicación el 24 de abril de 2004. Se aceptarán comentarios y/o discusiones hasta cinco meses después de su publicación.

(1) Instituto de Ingeniería, UNAM, Ciudad Universitaria, 04510 México, DF. jess@pumas.iingen.unam.mx; carlo_cisneros@hotmail.com; rgom@pumas.iingen.unam.mx 
la simplificación se enfoca al análisis de estructuras planas. En la actualidad, la proliferación de programas comerciales para análisis estructural permite llevar a cabo análisis de modelos estructurales tridimensionales de edificios sin mayor complicación. Sin embargo, este aspecto no ha facilitado el análisis por torsión de edificios. Uno de los factores fundamentales que lo complican es la llamada torsión accidental, ocasionada por la excentricidad accidental causada por diversas incertidumbres que afectan tanto al movimiento del suelo como a las propiedades y características que conforman a los materiales y elementos que integran a las estructuras.

Si se quisiera seguir al pie de la letra las recomendaciones de los reglamentos para incluir la torsión accidental explícitamente en los análisis estructurales de modelos tridimensionales de edificios, la cantidad de cálculos por hacer sería muy grande, a pesar de que se hicieran con la ayuda de una computadora. Una forma de resolver este problema consiste en elaborar programas de análisis de edificios que incluyan este aspecto automáticamente. Una forma más práctica de atacar este problema consiste en desarrollar técnicas que, utilizando programas de análisis estructural existentes, permitan incluir estos efectos. Actualmente existen diferentes propuestas que fundamentalmente buscan reducir lo laborioso del proceso del análisis por computadora de modelos tridimensionales de estructuras sometidas a torsión (Ávila, 1991; Goel y Chopra, 1992; Escobar et al., 2002, 2004).

En el presente trabajo se describen las bases teóricas que dan origen al análisis por torsión sísmica estática de estructuras de edificios. Para ello se estudia la distribución en edificios de las fuerzas cortantes entre los elementos resistentes de entrepiso debidas a torsión sísmica y se analizan los factores que afectan la excentricidad de diseño, entre ellas la excentricidad accidental. Se discuten las más recientes propuestas para realizar este análisis mediante el uso de programas comerciales de análisis estructural. También se propone y evalúa un nuevo procedimiento denominado Procedimiento Simplificado de Diseño, $P S D$, que, sin perder precisión en los cálculos, simplifica notablemente este tipo de análisis. Se demuestra que con el PSD propuesto, es suficiente un solo análisis de la estructura tridimensional, en cada una de sus direcciones ortogonales, para calcular las fuerzas de diseño en todos los elementos estructurales. Finalmente, se presentan dos ejemplos en los que se aplican las diferentes propuestas hechas y se discuten sus ventajas y desventajas.

\section{DISTRIBUCIÓN DE CORTANTES POR TORSIÓN}

El cortante total $V t o t_{i}$ en el $i$-ésimo elemento resistente del j-ésimo entrepiso del edificio será la suma algebraica del cortante directo $V d_{i}$, y el cortante por torsión $V t_{i}$, esto es

$V$ tot $_{i}=V d_{i}, \pm V t_{i}$

donde

$V d_{i}=\frac{k_{i}}{\Sigma k_{i}} V_{j}$ 
en esta ecuación $V_{j}$ es el cortante en el j-ésimo entrepiso. El cortante por torsión para la dirección $X$ en el i-ésimo elemento resistente será

$V t_{i}=\frac{M_{j}}{K_{\theta}} k_{i} y_{i}$

donde $K_{\theta}=\sum k_{i} x_{i}^{2}+\sum k_{i} y_{i}^{2}$, es la rigidez torsional del entrepiso, $x_{i}, y_{i}$ es la distancia a un punto de referencia, $M_{j}$. es el momento torsionante de entrepiso calculado como el producto del cortante de entrepiso y la excentricidad estructural o estática $e_{s}$. En edificios de varios pisos, el momento por torsión se puede calcular utilizando dos definiciones de excentricidad estática (Cheung y Tso, 1986, Tso, 1990), como se discute a continuación.

Excentricidad de piso. El momento de torsión $T_{j}$ del $j$-ésimo piso producido por la fuerza sísmica lateral $F_{j}$ y la excentricidad de piso $e_{s}$ para cada una de las direcciones ortogonales del edificio se calcula como

$T_{j}=F_{j} e_{s}$

donde la excentricidad de piso se calcula como la distancia entre el $C M$ y $C T$ correspondientes, esto es

$$
\begin{aligned}
& e_{s}=x_{C M}-x_{C T} \\
& e_{s}=y_{C M}-y_{C T}
\end{aligned}
$$

Las coordenadas del CM del j-ésimo piso se calculan como

$$
\begin{aligned}
& x_{C M}=\frac{\Sigma P_{i} y_{i}}{\Sigma P_{i}} \\
& y_{C M}=\frac{\Sigma P_{i} x_{i}}{\Sigma P_{i}}
\end{aligned}
$$

donde $P_{i}$ son las cargas verticales en el piso, y $x_{i}$ y $y_{i}$ son sus coordenadas respecto a un punto de referencia. El momento de torsión $M_{j}$ del entrepiso $j$, producido por el sismo en cada una de las direcciones $X$ y $Y$ se obtiene sumando los momentos de torsión de todos los pisos que se encuentran sobre éste

$$
M_{j}=\sum_{m=j}^{n} T_{m}
$$

donde $n$ es el número de entrepisos. 
Para calcular la posición del CT del piso se pueden utilizar los cortantes directos de los elementos resistentes. Estos se pueden obtener al aplicar las fuerzas sísmicas laterales $F x_{j}$ y $F y_{j}$, calculadas con un análisis sísmico estático o dinámico modal espectral, en los $C M$ correspondientes, permitiendo únicamente la traslación de los pisos. Así, las coordenadas del CT se calculan con las ecuaciones siguientes

$$
\begin{aligned}
x_{C T}= & \frac{\Sigma\left(V d y_{i j}-V d y_{i j-1}\right) x_{i}}{F y_{j}} \\
y_{C T}= & \frac{\Sigma\left(V d x_{i j}-V d x_{i j-1}\right) y_{i}}{F x_{j}}
\end{aligned}
$$

donde $V d x_{i j}, V d y_{i j}$ son los cortantes directos del $i$-ésimo elemento resistente, y $x_{i}$ y $y_{i}$ son sus coordenadas respecto a un punto de referencia en las direcciones $X$ y $Y$ en el entrepiso $j$, respectivamente.

Excentricidad de entrepiso. El momento de torsión $M_{j}$ del $j$-ésimo entrepiso, para cada una de las direcciones ortogonales $X$ y $Y$, se obtiene directamente como el producto de la fuerza cortante $V_{j}$ y la excentricidad de entrepiso $e_{s}$, esto es

$$
M_{j}=V_{j} e_{s}
$$

La excentricidad de entrepiso para cada una de las direcciones ortogonales $X$ y $Y$ y se calcula como la distancia entre el centro de cortantes, $C C$, y el centro de rigideces $C R$ del entrepiso como

$$
\begin{aligned}
& e_{s}=x_{C C}-x_{C R} \\
& e_{s}=y_{C C}-y_{C R}
\end{aligned}
$$

El CC depende de la distribución de las fuerzas laterales en el edificio y sus coordenadas se calculan como

$$
\begin{aligned}
& x_{C C}=\frac{\sum F y_{j} x_{C M}}{V y_{j}} \\
& y_{C C}=\frac{\sum F x_{j} y_{C M}}{V x_{j}}
\end{aligned}
$$

donde $F x_{j}$ y $F y_{j}$, son las fuerzas sísmicas laterales aplicadas en los $C M$ permitiendo únicamente la traslación de los pisos; $V x_{j}$ y $V y_{j}$ son los cortantes del entrepiso en las direcciones $X$ y $Y$ en el entrepiso $j$, respectivamente. 


$$
\begin{aligned}
& x_{C R}=\frac{\Sigma\left(V d y_{i} x_{i}\right)}{V y_{j}} \\
& y_{C R}=\frac{\Sigma\left(V d x_{i} y_{i}\right)}{V x_{j}}
\end{aligned}
$$

o también con las rigideces de los elementos resistentes como

$$
\begin{aligned}
& x_{C R}=\frac{\sum\left(k_{i} x_{i}\right)}{\sum k_{i}} \\
& y_{C R}=\frac{\sum\left(k_{i} y_{i}\right)}{\sum k_{i}}
\end{aligned}
$$

En un estudio previo (Escobar et al., 2004), se aplicaron los conceptos de excentricidad de piso y de entrepiso al edifico de cinco pisos estudiado por Bazán y Meli (2000) de la Fig. 1. Los resultados obtenidos muestran que los valores de los momentos de torsión calculados con las dos definiciones de excentricidad estática son prácticamente iguales entre sí; las diferencias observadas se pueden atribuir a las operaciones aritméticas que intervienen en los cálculos.

\section{DISEÑO POR TORSIÓN SÍSMICA ESTÁTICA}

Para considerar el efecto de la torsión sísmica, el Reglamento de Construcciones para el Distrito Federal, RCDF, permite llevar a cabo un análisis estático de las estructuras de edificios (RCDF-95, 1995). Sin embargo, las solicitaciones que se obtienen de este análisis son diferentes de las que resultan de un análisis dinámico tridimensional de las mismas. Para tomar en cuenta este efecto el RCDF permite utilizar factores de amplificación de la excentricidad estática.

Por otra parte, los momentos de torsión en edificios reales difieren de los obtenidos en un análisis dinámico debido a factores no incluidos explícitamente (Rosenblueth, 1979). La manera de considerarlos en el diseño sísmico de estructuras es tomando en cuenta un momento de torsión adicional, denominado momento por torsión accidental, el cual se calcula desplazando de su posición original, a la fuerza cortante que actúa en el entrepiso, una cierta cantidad denominada excentricidad accidental. Esta se expresa como un porcentaje de la dimensión máxima de la planta de la estructura que es perpendicular a la dirección del sismo. 


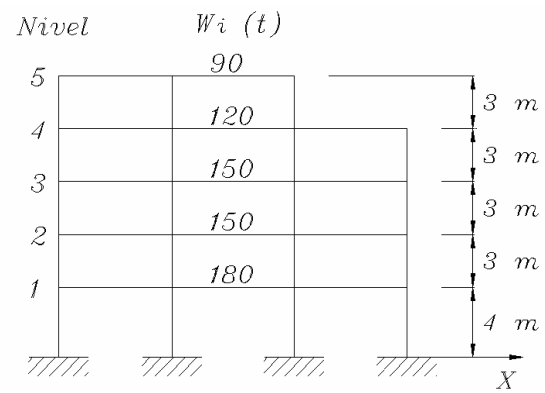

a) Elevación

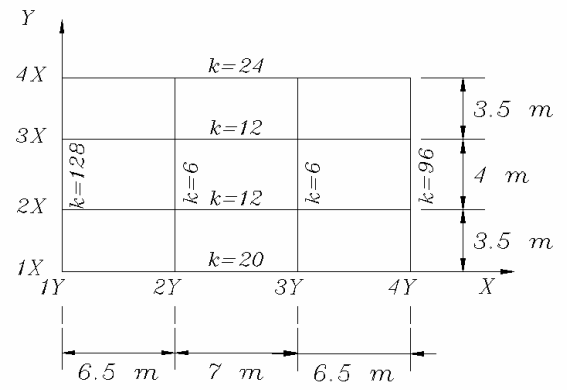

b) Entrepiso 1 a 3

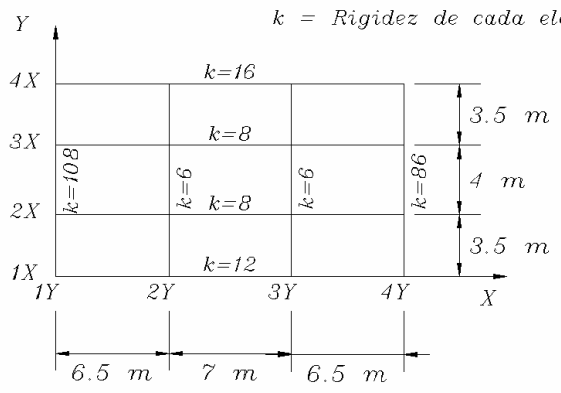

c) Entrepiso 4

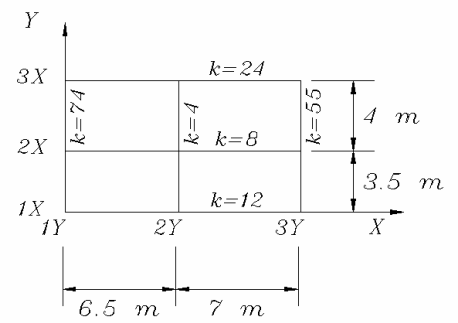

d) Entrepiso 5

Figura 1. Edificio de cinco pisos estudiado por Bazán y Meli (2000).

Así, si se utiliza el RCDF (RCDF-95, 1995), el cálculo de las fuerzas de diseño de los elementos resistentes del j-ésimo entrepiso, requiere del cálculo del cortante directo de diseño, $V d_{i}$, con la ecuación (2), mientras que la fuerza cortante producida por la torsión sísmica se obtiene del momento torsionante originado por la aplicación del cortante de entrepiso multiplicado por una excentricidad de diseño ed. Esta última que puede adoptar los siguientes dos valores

$e d=\alpha e_{s}+\beta b$

$e d=\delta e_{s}-\beta b$

donde, $\alpha=1.5$ y $\delta=1.0$, son los factores de amplificación dinámica; $\beta=0.1$ es el factor de excentricidad accidental, y $b$ es la dimensión máxima en planta de la estructura, perpendicular a la dirección de excitación sísmica que se analiza. De las ecuaciones (14) se utiliza como excentricidad de diseño aquella que ocasione los efectos más desfavorables en cada uno de los elementos resistentes.

Por lo tanto, las fuerzas cortantes de diseño debidas a la torsión del entrepiso tomando en cuenta la excentricidad de diseño, se obtienen de la distribución del momento torsionante de acuerdo con la contribución de los elementos estructurales a la rigidez torsional del mismo, esto es 


$$
V t_{i}=\frac{k_{i} x_{i}}{K_{\theta}} V_{j} e d_{i}
$$

Al considerar los efectos anteriores, es posible analizar las estructuras de edificios tomando en cuenta únicamente la traslación de los entrepisos. Así, de acuerdo con las ecuaciones anteriores, el cortante total que deberá resistir el i-ésimo elemento resistente de un entrepiso será

$$
\text { Vtot }_{i}=\frac{k_{i}}{\Sigma k_{i}} V_{j} \pm \frac{k_{i} x_{i}}{K_{\theta}} V_{j} e d_{i}
$$

Para la aplicación de esta ecuación se debe tomar en cuenta lo especificado en el párrafo 8.6 de las Normas Técnicas Complementarias para Diseño por Sismo del RCDF, NTCDS-95 (NTC-95, 1995), en el que se establece que la excentricidad de diseño ed en cada dirección no será menor que la mitad del máximo valor de $e_{s}$ para los entrepisos que se hallan abajo del que se considera, ni se tomará el momento por torsión de ese entrepiso menor que la mitad del máximo calculado para los entrepisos que están arriba del considerado.

Por otro lado, también se deberán tener presente los efectos bidireccionales del sismo. Las NTCDS-95 los toman en cuenta estableciendo que cada sección crítica de un edificio debe resistir la suma vectorial de los efectos (desplazamientos y fuerzas internas) de un componente del movimiento del terreno con 30 \% de los del otro, en adición a los efectos de las fuerzas gravitatorias.

Como se puede apreciar en las ecuaciones anteriores, para incluir el efecto de la torsión, es posible llevar a cabo un análisis estático de las estructuras de edificios, considerando que las fuerzas sísmicas actúan en el centro de masas de los pisos, y que los momentos de torsión correspondientes son distribuidos entre los elementos resistentes.

En la Fig. 2 se presenta esquemáticamente el efecto de la torsión sísmica en los elementos resistentes de un edificio de varios pisos.

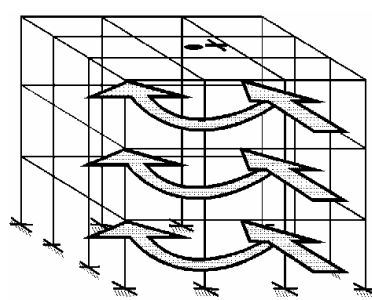

a) Efecto de la torsion sísmica

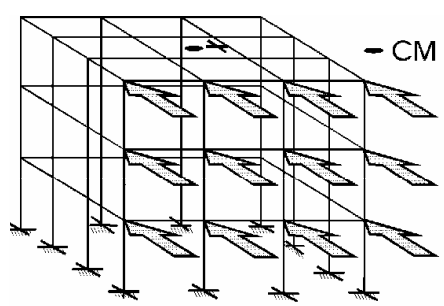

b) Cortante directo $V d_{i}$

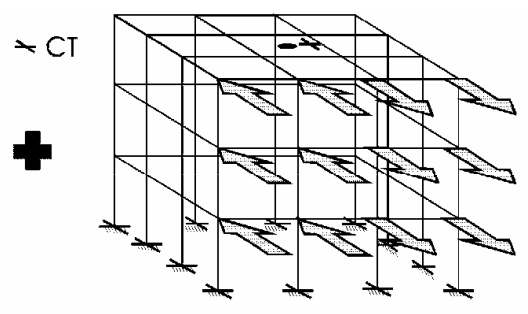

c) Cortante por torsión $V t_{i}$

Figura 2. Representación esquemática del efecto de la torsión sísmica en los elementos resistentes de un edificio. 


\section{PROCEDIMIENTOS PARA DISTRIBUIR LAS FUERZAS SÍSMICAS POR TORSIÓN}

Se presentan diferentes procedimientos para distribuir las fuerzas cortantes por torsión. Todos ellos se basan en el cálculo de la fuerza horizontal aplicada en cada nivel, obtenida mediante un análisis sísmico estático o dinámico modal espectral.

En todos los métodos presentados, se requiere que para cada elemento resistente se calculen los cortantes debidos al $100 \%$ de las fuerzas sísmicas en la dirección $X$ más $30 \%$ de las fuerzas sísmicas en la dirección $Y$ y viceversa. Para el diseño rige el mayor de estos resultados.

\section{Método de Rosenblueth-Esteva}

Para distribuir las fuerzas cortantes por torsión, Rosenblueth y Esteva (1962) propusieron el siguiente procedimiento.

1. Para las dos direcciones principales en que se efectuará el análisis, calcular por estática la línea de acción del cortante sísmico en cada entrepiso, $C M$.

2. Con las rigideces de los elementos de cada entrepiso, o con los cortantes directos, calcular la posición del $C T$ de cada entrepiso.

3. La fuerza cortante total que debe ser soportada por cada uno de los elementos resistentes de cada entrepiso será igual al valor más desfavorable de las fuerzas calculadas como

$$
V t^{\prime} t_{i(+)}=V d_{i}+V t_{i} ; \quad \text { ó } \quad V t o t_{i(-)}=V d_{i}-V t_{i}
$$

4. Las fuerzas de diseño actuantes en los elementos estructurales se calculan haciendo un análisis individual en el plano de cada uno de los elementos resistentes con base en la fuerza cortante total calculada en el paso anterior.

\section{Método de Ávila}

Este método es el primero en nuestro país que ha sido planteado con el objetivo de ser aplicado utilizando programas de análisis estructural comerciales. Consiste de los siguientes pasos (Ávila, 1991):

1. Calcular los valores de las coordenadas del $C M$ de cada uno de los pisos.

2. Calcular los cortantes directos en los elementos estructurales. Esto se puede realizar aplicando estáticamente, las fuerzas calculadas en el análisis sísmico estático, en $C M$ de cada uno de los pisos del modelo tridimensional de la estructura impidiendo los giros de las losas alrededor de un eje vertical.

3. Con los cortantes directos y las ecuaciones (21) se calculan las coordenadas de CT de cada uno de los entrepisos. 
4. Con las coordenadas de $C M$ y $C T$ de cada uno de los pisos se calcula la excentricidad estructural.

5. Con las ecuaciones

$$
\begin{aligned}
& x^{*}{ }_{C M 1}=x_{C T}+E d_{1} ; \quad y, \quad x^{*}{ }_{C M 2}=x_{C T}+E d_{2} \\
& y^{*}{ }_{C M 1}=x_{C T}+E d_{1} ; \quad y, \quad y_{C M 2}^{*}=x_{C T}+E d_{2}
\end{aligned}
$$

donde

$$
E d_{1}=1.5 e_{s}+0.1 b\left(\text { signo } e_{s}\right) ; \quad \mathrm{y}, \quad E d_{2}=e_{s}-0.1 b\left(\text { signo } e_{s}\right)
$$

se calculan las coordenadas modificadas de $C M$ de cada piso para cada una de las direcciones de análisis $X$ y $Y$.

6. Para cada una de las dos direcciones ortogonales de la estructura, se realizan dos análisis estructurales permitiendo traslación y rotación de los entrepisos. En cada uno de ellos se deberá mover la posición nominal de CM una distancia calculada con las ecuaciones (18) y (19) anteriores.

7. Las fuerzas que deberán resistir los elementos estructurales al considerar la torsión sísmica serán aquellos que presenten los valores máximos obtenidos de los análisis estructurales realizados en el paso anterior para cada una de las direcciones ortogonales del edificio.

\section{Método de Goel-Chopra}

Este método (Goel y Chopra, 1992) es muy similar al propuesto por Bazán (1978). Ambos tienen la particularidad de que no requieren del cálculo del $C T$ de los pisos ni del $C R$ de los entrepisos. A diferencia del método propuesto por Bazán en 1978, la aplicación de método de Goel-Chopra se lleva a cabo utilizando programas comerciales de análisis estructural. Consiste de los pasos siguientes

1. Calcular los valores de las coordenadas de $C M$ de cada uno de los pisos.

2. Para cada una de las direcciones ortogonales de la estructura, en $C M$ de cada uno de los pisos del modelo tridimensional se aplican las fuerzas calculadas en el análisis sísmico estático de la misma impidiendo los giros de las losas alrededor de un eje vertical. A los resultados obtenidos de este análisis se les denominará $V 1$.

3. Para cada una de las direcciones ortogonales realizar un análisis de la estructura similar al anterior pero ahora permitiendo el giro de las losas de los entrepisos alrededor de un eje vertical. A los resultados obtenidos de este análisis se les denominará V2.

4. Aplicando un momento torsionante debido a la excentricidad accidental en cada uno de los entrepisos realizar un análisis de la estructura similar al anterior, esto es 
$M_{j}=\beta b V_{j}$

Para el RCDF vigente, $\beta=0.1$. A los resultados obtenidos de este análisis se les denominará $V 3$.

5. El efecto total debido a las fuerzas laterales y de torsión en los elementos resistentes se obtendrá al combinar los resultados obtenidos en los análisis anteriores para cada una de las direcciones ortogonales aplicando las ecuaciones siguientes

$V t 1=(1-\alpha) V 1+\alpha V 2 \pm V 3$

$V t 2=(1-\delta) V 1+\delta V 2-V 3$

Para el RCDF-95, $\alpha=1.5$, y $\delta=1.0$, entonces, las ecuaciones anteriores serán

$V t 1=-(0.5) V 1+(1.5) V 2 \pm V 3$

$V t 2=V 2-V 3$

6. De los resultados obtenidos de las dos combinaciones anteriores se elegirán para diseño, para cada una de las direcciones ortogonales de la estructura, los valores máximos de las fuerzas en los elementos estructurales.

\section{Procedimiento Simplificado de Diseño PSD}

El procedimiento simplificado de diseño por torsión sísmica estática, PSD (Escobar, et al., 2002, 2004) utiliza factores de amplificación por torsión. En su planteamiento se buscó que pudiera ser aplicado utilizando programas comerciales de análisis estructural. Consta de los siguientes pasos

1. Calcular las coordenadas de $C M$ de cada uno de los pisos.

2. Para las dos direcciones ortogonales de la estructura se calculan los cortantes directos en los elementos resistentes aplicando, estáticamente, las fuerzas obtenidas del análisis sísmico estático, en $C M$ de los pisos del modelo tridimensional de la misma impidiendo los giros de las losas alrededor de un eje vertical.

3. Calcular las coordenadas de $C R$ y $C C$ de cada uno de los entrepisos.

4. Clasificar a los elementos resistentes como rígidos si están localizados del mismo lado de $C R$ con respecto a $C C$, y como flexibles en caso contrario.

5. Calcular la excentricidad estática de cada uno de los entrepisos.

6. Calcular los factores de amplificación por torsión FAT de los elementos resistentes del jésimo entrepiso como 
FATf $_{i}=1+\frac{\zeta_{i}}{\rho^{2}}(\beta+\alpha e) ; \mathrm{y}, \quad$ FATr $_{i}=1+\frac{\zeta_{i}}{\rho^{2}}(\beta-\delta e)$

Para el caso del RCDF, con $\alpha=1.5, \beta=0.1$ y $\delta=1.0$ se tiene que

$$
\text { FATf }_{i}=1+\frac{\zeta_{i}}{\rho^{2}}(0.1+1.5 e) ; \quad \mathrm{y}, \quad \text { FATr }_{i}=1+\frac{\zeta_{i}}{\rho^{2}}(0.1-e)
$$

donde $F_{A T f_{i}}$ y FATr son los factores de amplificación por torsión del i-ésimo elemento flexible y rígido respectivamente; $\zeta_{i}=x_{i} / b$ es la posición, $x_{i}$, con respecto a $C R$ normalizada del i-ésimo elemento resistente; $e=\left|e_{s} b\right|$ es el valor absoluto de la excentricidad estática normalizada; y $\rho$ es el radio de giro normalizado del $j$-ésimo entrepiso. Este parámetro se puede calcular para cada una de las direcciones $X$ y $Y$ de la estructura como

$$
\begin{aligned}
& \rho_{x j}=\frac{1}{b_{x j}} \sqrt{\frac{\sum V d y_{i} x_{i}^{2} / d y_{j}+\sum V d x_{i} y_{i}^{2} / d x_{j}}{\sum V d x_{i} / d x_{j}}} \\
& \rho_{y j}=\frac{1}{b_{y j}} \sqrt{\frac{\sum V d y_{i} x_{i}^{2} / d y_{j}+\sum V d x_{i} y_{i}^{2} / d x_{j}}{\sum V d y_{i} / d y_{j}}}
\end{aligned}
$$

donde $d x_{j}$ y $d y_{j}$ son los desplazamientos relativos del j-ésimo entrepiso obtenidos del análisis estructural estático realizado en el paso 2 para determinar los cortantes directos en los elementos estructurales.

7. Obtener las fuerzas totales de diseño (fuerzas cortantes, axiales, momentos flexionantes, etc.) en los elementos estructurales, multiplicando las fuerzas producidas en ellos por los cortantes directos calculados en el paso 2 anterior, por los FAT correspondientes, esto es

$$
\operatorname{Vtot}_{i}=\operatorname{FATf}_{i}\left(V d_{i}\right) ; \quad \mathrm{y} \quad \operatorname{Vtot}_{i}=\operatorname{FATr}_{i}\left(V d_{i}\right)
$$

\section{EJEMPLOS DE APLICACIÓN}

Se presentan ejemplos de aplicación de los métodos de distribución de las fuerzas laterales descritos. En todos los casos, las fuerzas cortantes de entrepiso se calculan a partir de un análisis sísmico estático considerando un sistema de fuerzas equivalentes obtenidas con un espectro de diseño sísmico. 


\section{Estructura de un piso}

Se diseñará por torsión la estructura de un piso mostrada en la Fig. 3. En esta figura también se muestran su periodo fundamental de vibración y otros datos. Se considera que la estructura estará ubicada en la Zona III del DF. Sólo se supondrá excitación en dirección $Y$. En el Apéndice A se presentan los cálculos correspondientes.

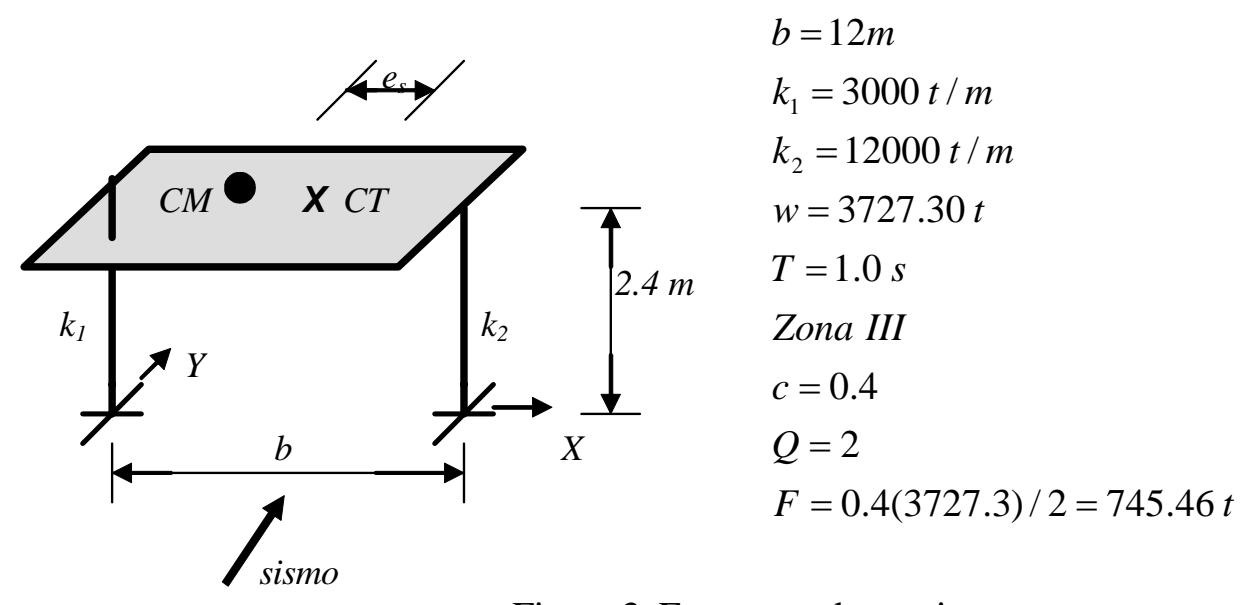

Figura 3. Estructura de un piso.

Método de Rosenblueth-Esteva. En la Tabla 1 se anotan los cálculos y resultados obtenidos de la aplicación de este método.

Tabla 1.Cortantes totales (t) obtenidos con el método de Rosenblueth-Esteva.

\begin{tabular}{|c|c|c|c|c|c|c|c|c|}
\hline Elemento & $\boldsymbol{k}_{\boldsymbol{i}}$ & $\boldsymbol{x}_{\boldsymbol{i}}$ & $\boldsymbol{k}_{\boldsymbol{i}} \boldsymbol{x}_{\boldsymbol{i}}$ & $\boldsymbol{k}_{\boldsymbol{i}} \boldsymbol{x}_{\boldsymbol{i}}^{\mathbf{2}}$ & $\boldsymbol{V d}_{\boldsymbol{i}}$ & $\boldsymbol{V t}_{\boldsymbol{i}(+)}$ & $\boldsymbol{V t}_{\boldsymbol{i}(-)}$ & $\boldsymbol{V t o t}_{\boldsymbol{i}}$ \\
\hline 1 & 3000 & 9.6 & 28800 & 276480 & 149.10 & 410.0 & 149.1 & 559.10 \\
\hline 2 & 12000 & 2.4 & 28800 & 69120 & 596.37 & 410.0 & 149.1 & 447.27 \\
\hline Suma & 15000 & & & 345600 &
\end{tabular}

Método de Ávila. Algunos de los cálculos necesarios para la aplicación de este método se presentan en la Tabla 2. Al hacer los dos análisis de la estructura con los CM localizados en las nuevas coordenadas, se obtienen los resultados mostrados en la Tabla 3.

Tabla 2. Coordenadas y valores de la excentricidad de diseño. Estructura de un piso.

\begin{tabular}{|c|c|c|c|c|c|}
\hline Piso & $\boldsymbol{X}_{\boldsymbol{C M}}$ & $\boldsymbol{X}_{\boldsymbol{C T}}$ & $\boldsymbol{e}_{\mathbf{c}}$ & $\boldsymbol{E d}_{1}$ & $\boldsymbol{E d}_{\mathbf{2}}$ \\
\hline 1 & 6.0 & 9.6 & -3.6 & -6.6 & -2.4 \\
\hline
\end{tabular}

Tabla 3. Nuevas coordenadas del CM y cortantes totales (t). Método de Ávila. Estructura de un piso.

\begin{tabular}{|c|c|c|c|c|}
\hline Análisis & Elemento & $\boldsymbol{X}^{*} \mathbf{C M}$ & $\boldsymbol{V d}_{\boldsymbol{i}}+\boldsymbol{V t}_{\boldsymbol{i}}$ & $\boldsymbol{V t o t}_{\boldsymbol{i}}$ \\
\hline \multirow{2}{*}{1} & 1 & 3.0 & 554.48 & 554.48 \\
\cline { 2 - 5 } & 2 & & 190.98 & --- \\
\hline \multirow{2}{*}{2} & 1 & 7.2 & 296.51 & --- \\
\cline { 2 - 5 } & 2 & & 448.95 & 448.95 \\
\hline
\end{tabular}


Método de Goel-Chopra. Para cada una de las condiciones de carga (V1, V2 y V3) se requiere hacer tres análisis de la estructura. Los cortantes obtenidos en los elementos resistentes se muestran en la Tabla 4. Al hacer las dos combinaciones de carga especificadas por el método se obtienen los valores del cortante total en los elementos que se anotan en la última columna de la Tabla 4.

Tabla 4.Cortantes (t) obtenidos con el método de Goel-Chopra. Estructura de un piso.

\begin{tabular}{|c|c|c|c|c|c|c|}
\hline Elemento & $\boldsymbol{V 1}$ & $\boldsymbol{V} \mathbf{2}$ & $\boldsymbol{V 3}$ & $\boldsymbol{V t 1}$ & $\boldsymbol{V t} 2$ & Vtot $_{\boldsymbol{i}}$ \\
\hline 1 & 148.92 & 370.21 & 73.71 & 554.57 & 296.5 & 554.57 \\
\hline 2 & 596.54 & 375.25 & -73.71 & 190.01 & 448.96 & 448.96 \\
\hline
\end{tabular}

Procedimiento Simplificado de Diseño PSD. Al aplicar las ecuaciones (29) se obtienen los cortantes totales a partir de los cortantes directos en los elementos estructurales. En la Tabla 5 se anotan los cálculos y resultados obtenidos con la aplicación del PSD.

Tabla 5. Cortantes totales (t), obtenidos con el PSD. Estructura de un piso ( $f=$ elemento flexible, $r$ =elemento rígido).

\begin{tabular}{|c|c|c|c|c|}
\hline Elemento, & $\boldsymbol{x}_{\boldsymbol{i}}$ & $\zeta_{\mathbf{i}}$ & $\boldsymbol{F A T}_{\boldsymbol{i}}$ & Vtot $_{\boldsymbol{i}}$ \\
\hline $1, \mathrm{f}$ & 9.6 & 0.8 & 3.75 & 559.12 \\
\hline $2, \mathrm{r}$ & 2.4 & 0.2 & 0.75 & 447.27 \\
\hline
\end{tabular}

\section{Edificio de cinco pisos}

Se analiza el edificio de cinco pisos (Fig. 1), estudiado por Bazán y Meli (2000). Las fuerzas laterales para las direcciones $X$ y $Y$ se presentan en la Tabla 6. Por razones de espacio, la aplicación de los métodos se ilustra únicamente para el piso cinco del edificio. En el Apéndice B se presentan los cálculos correspondientes.

Tabla 6. Fuerzas y cortantes en el edificio de cinco pisos.

\begin{tabular}{|c|c|c|c|c|}
\hline $\begin{array}{c}\text { Piso } \\
\text { Entrepiso }\end{array}$ & $F x_{i}(t)$ & $F y_{i}(t)$ & $V x_{j}(t)$ & $V y_{j}(t)$ \\
\hline 5 & 23.77 & 47.54 & & \\
\hline 5 & & & 23.77 & 47.54 \\
\hline 4 & 25.75 & 51.50 & & \\
\hline 4 & & & 49.52 & 99.04 \\
\hline 3 & 24.76 & 49.52 & & \\
\hline 3 & & & 74.28 & 148.56 \\
\hline 2 & 17.33 & 34.67 & & \\
\hline 2 & & & 91.61 & 183.23 \\
\hline 1 & 11.89 & 23.77 & & \\
\hline 1 & & & 103.50 & 207.00 \\
\hline
\end{tabular}

En este caso, además de los cortantes totales en los elementos resistentes, como resultados se presentan fuerzas de diseño (fuerzas axiales y momentos flexionantes) de diferentes elementos estructurales. 
Método de Rosenblueth-Esteva. En las Tablas 7, 8 y 17 se anotan los cálculos y resultados obtenidos de la aplicación de este método para las direcciones de análisis $X$ y $Y$, respectivamente. En la Tabla 18 se anotan los resultados para las fuerzas de diseño de algunos de los elementos estructurales obtenidos después de realizar los análisis estructurales correspondientes de los elementos resistentes planos (en este caso 8 análisis de igual número de modelos estructurales).

Tabla 7. Distribución de la fuerza cortante (t) por sismo entre los elementos resistentes. Método de Rosenblueth-Esteva, sismo en dirección $X$ del piso cinco del edificio de cinco pisos.

\begin{tabular}{|c|c|c|c|c|c|c|c|c|c|c|}
\hline \multirow[t]{2}{*}{ Elemento } & \multirow[t]{2}{*}{$\boldsymbol{k}_{i}$} & \multirow[t]{2}{*}{$y_{i}$} & \multirow[t]{2}{*}{$k_{i} y_{i}$} & \multirow[t]{2}{*}{$y_{t i}$} & \multirow[t]{2}{*}{$k_{i} y_{t i}$} & \multirow[t]{2}{*}{$k_{i} y_{t i}^{2}$} & \multirow[t]{2}{*}{$V d_{i}$} & \multicolumn{2}{|c|}{$V_{t}$} & \multirow[t]{2}{*}{ Vtot $_{i}$} \\
\hline & & & & & & & & $V t_{i(+)}$ & $V t_{i(-)}$ & \\
\hline $1 X$ & 1200 & 0 & 0 & -4.73 & -5676.0 & 26847.48 & 6.48 & 0.49 & 0.05 & 6.97 \\
\hline $2 X$ & 800 & 3.5 & 2800 & -1.23 & -984.0 & 1210.32 & 4.32 & 0.08 & 0.01 & 4.41 \\
\hline $3 X$ & 2400 & 7.5 & 18000 & 2.77 & 6648.0 & 18414.96 & 12.97 & 0.57 & 0.06 & 12.91 \\
\hline Suma & 4400 & & 20800 & & & 46472.76 & 23.77 & & & \\
\hline
\end{tabular}

Tabla 8. Distribución de la fuerza cortante (t) por sismo entre los elementos resistentes. Método de Rosenblueth-Esteva, sismo en dirección $Y$ del piso cinco del edificio de cinco pisos.

\begin{tabular}{|c|c|c|c|c|c|c|c|c|c|c|}
\hline \multirow[t]{2}{*}{ Elemento } & \multirow[t]{2}{*}{$\boldsymbol{k}_{i}$} & \multirow[t]{2}{*}{$x_{i}$} & \multirow[t]{2}{*}{$k_{i} x_{i}$} & \multirow[t]{2}{*}{$x_{t i}$} & \multirow[t]{2}{*}{$k_{i} x_{t i}$} & \multirow[t]{2}{*}{$k_{i} x_{t i}^{2}$} & \multirow[t]{2}{*}{$V d_{i}$} & \multicolumn{2}{|c|}{$V t$} & \multirow[t]{2}{*}{ Vtot $_{i}$} \\
\hline & & & & & & & & $V t_{i(+)}$ & $V t_{i(-)}$ & \\
\hline $1 Y$ & 7400 & 0 & 0 & -5.78 & -42772.0 & 247222.16 & 26.45 & 9.19 & 1.24 & 27.69 \\
\hline $2 Y$ & 400 & 6.5 & 2600 & 0.72 & 288.0 & 207.36 & 1.43 & 0.06 & 0.01 & 1.49 \\
\hline $3 Y$ & 5500 & 13.5 & 74250 & 7.72 & 42460.0 & 327731.2 & 19.66 & 9.12 & 1.23 & 28.78 \\
\hline Suma & 13300 & & 76850 & & & 575220.72 & 47.54 & & & \\
\hline
\end{tabular}

Método de Ávila. La idea fundamental de este método se basa en el cambio de posición de CM. En las Tablas 9 y 10 se presentan algunos de los cálculos necesarios para su aplicación. Al hacer los dos análisis para cada una de las direcciones ortogonales de la estructura con los CM localizados en las nuevas coordenadas, se obtienen los resultados mostrados en las Tablas 11, 17 y 18.

Tabla 9. Cálculos para la aplicación del método de Ávila. Edificio de cinco pisos.

\begin{tabular}{|c|c|c|c|c|c|c|c|c|c|c|c|c|}
\hline \multirow[t]{2}{*}{ Piso } & \multirow[t]{2}{*}{$x_{C M}$} & \multirow[t]{2}{*}{$y_{C M}$} & \multirow[t]{2}{*}{$x_{C T}$} & \multirow[t]{2}{*}{$y_{C T}$} & \multirow[t]{2}{*}{$\boldsymbol{e}_{s x}$} & \multirow[t]{2}{*}{$\boldsymbol{e}_{s x}$} & \multicolumn{3}{|c|}{ Sismo en dirección $X$} & \multicolumn{3}{|c|}{ Sismo en dirección $Y$} \\
\hline & & & & & & & $b$ & $E d_{1}$ & $E d_{2}$ & $b$ & $E d_{1}$ & $E d_{2}$ \\
\hline 5 & 6.75 & 3.75 & 5.87 & 4.73 & 0.88 & -0.98 & -7.5 & -2.22 & -0.23 & 13.5 & 2.67 & -0.47 \\
\hline 4 & 9.2 & 5.5 & 11.76 & 7.17 & -2.56 & -1.67 & -11 & -3.605 & -0.57 & -20 & -5.84 & -0.56 \\
\hline 3 & 9.2 & 5.5 & 8.07 & 5.47 & 1.13 & 0.03 & 11 & 1.145 & -1.07 & 20 & 3.69 & -0.87 \\
\hline 2 & 9.2 & 5.5 & 8.64 & 5.82 & 0.56 & -0.32 & -11 & -1.58 & 0.78 & 20 & 2.81 & -1.46 \\
\hline 1 & 8.5 & 6.3 & 8.64 & 5.82 & -0.14 & 0.48 & 11 & 1.82 & -0.62 & -20 & -2.18 & 1.88 \\
\hline
\end{tabular}

Método de Goel-Chopra. En este caso se requiere hacer seis análisis de la estructura para las tres condiciones de carga (V1, V2 y V3). Los cortantes obtenidos en los elementos resistentes, en 
cada una de las direcciones ortogonales $X$ y $Y$ de la estructura se muestran en la Tabla 12 . Al hacer las dos combinaciones de carga especificadas en las ecuaciones (24) y (25) para los elementos, se obtiene el cortante total que se anota en la última columna de la misma Tabla y en las 17 y 18 para los elementos estructurales.

Tabla 10. Nuevas coordenadas del CM (m) para la aplicación del método de Ávila. Edificio de cinco pisos.

\begin{tabular}{|c|c|c|c|c|}
\hline \multirow[t]{2}{*}{ Piso } & \multicolumn{2}{|c|}{$E d_{1}$} & \multicolumn{2}{|c|}{$E d_{2}$} \\
\hline & $x^{*}{ }_{C M 1}$ & $y^{*}{ }_{C M 1}$ & $x^{*}{ }_{C M 2}$ & $y^{*}{ }_{C M 2}$ \\
\hline 5 & 8.54 & 2.51 & 5.40 & 4.50 \\
\hline 4 & 5.92 & 3.57 & 11.2 & 6.60 \\
\hline 3 & 11.77 & 6.62 & 7.20 & 4.40 \\
\hline 2 & 11.47 & 4.24 & 7.20 & 6.60 \\
\hline 1 & 6.44 & 7.64 & 10.5 & 5.20 \\
\hline
\end{tabular}

Tabla 11. Cortantes totales (t) obtenidos con el método de Ávila. Edificio de cinco pisos.

\begin{tabular}{|c|c|c|c|c|c|c|c|}
\hline \multicolumn{4}{|c|}{ Sismo en dirección $\boldsymbol{X}$} & \multicolumn{4}{c|}{ Sismo en dirección $\boldsymbol{V}$} \\
\hline Análisis & Elemento & $\boldsymbol{V d}_{\boldsymbol{i}}+\boldsymbol{V t}_{\boldsymbol{i}}$ & $\boldsymbol{V t o t}_{\boldsymbol{i}}$ & Análisis & Elemento & $\boldsymbol{V d}_{\boldsymbol{i}}+\boldsymbol{V t}_{\boldsymbol{i}}$ & $\boldsymbol{V t o t}_{\boldsymbol{i}}$ \\
\hline \multirow{3}{*}{$\begin{array}{c}1 \\
\left(E d_{1}\right)\end{array}$} & $1 X$ & 6.66 & 6.66 & 3 & $1 Y$ & 26.67 & 26.67 \\
\cline { 2 - 8 } & $2 X$ & 4.35 & --- & $\left(E d_{1}\right)$ & $2 Y$ & 1.44 & --- \\
\cline { 2 - 8 } & $3 X$ & 12.78 & --- & & $3 Y$ & 19.44 & --- \\
\hline \multirow{2}{*}{2} & $1 X$ & 6.51 & --- & 4 & $1 Y$ & 23.1 & --- \\
\cline { 2 - 8 }$\left(E d_{2}\right)$ & $2 X$ & 4.36 & 4.36 & $\left(E d_{2}\right)$ & $2 Y$ & 1.47 & 1.47 \\
\cline { 2 - 8 } & $3 X$ & 12.96 & 12.96 & & $3 Y$ & 22.98 & 22.98 \\
\hline
\end{tabular}

Tabla 12. Cortantes (t) obtenidos con el método de Goel-Chopra. Edificio de cinco pisos.

\begin{tabular}{|c|c|c|c|c|c|c|c|}
\hline Dirección & Elemento & $\mathbf{V 1}$ & $\boldsymbol{V} \mathbf{2}$ & $\boldsymbol{V 3}$ & $\boldsymbol{V t 1}$ & $\boldsymbol{V t} \mathbf{2}$ & Vtot $_{\boldsymbol{i}}$ \\
\hline \multirow{3}{*}{$X$} & $1 X$ & 6.48 & 6.57 & 0.09 & 6.71 & 6.48 & 6.71 \\
\cline { 2 - 8 } & $2 X$ & 4.32 & 4.32 & 0.018 & 4.34 & 4.30 & 4.34 \\
\cline { 2 - 8 } & $3 X$ & 12.96 & 12.87 & 0.12 & 12.95 & 12.75 & 12.95 \\
\hline \multirow{3}{*}{$Y$} & $1 Y$ & 26.45 & 25.14 & 0.84 & 25.49 & 24.3 & 25.49 \\
\cline { 2 - 8 } & $2 Y$ & 1.43 & 1.47 & 0.005 & 1.49 & 1.47 & 1.49 \\
\cline { 2 - 8 } & $3 Y$ & 19.66 & 20.97 & 0.84 & 22.32 & 20.13 & 22.32 \\
\hline
\end{tabular}

Tabla 13. Cortantes en los elementos resistentes obtenidos con el PSD. Edificio de cinco pisos ( $f$ =elemento flexible, $\mathrm{r}=$ =elemento rígido).

\begin{tabular}{|c|c|c|c|c|c|c|c|}
\hline \multicolumn{4}{|c|}{ Sismo en dirección $\boldsymbol{~}$} & \multicolumn{3}{c|}{ Sismo en dirección $\boldsymbol{Y}$} \\
\hline $\begin{array}{c}\text { Elemento } \\
\text { tipo }\end{array}$ & $\zeta_{\boldsymbol{I}}$ & $\boldsymbol{F A \boldsymbol { T } _ { \boldsymbol { i } }}$ & $\boldsymbol{V t o t}_{\boldsymbol{i}}(\boldsymbol{t})$ & $\begin{array}{c}\text { Elemento } \\
\text { Tipo }\end{array}$ & $\zeta_{\boldsymbol{I}}$ & $\boldsymbol{F A T _ { \boldsymbol { i } }}$ & $\boldsymbol{V t o t}_{\boldsymbol{i}}(\boldsymbol{t})$ \\
\hline $1 X, \mathrm{f}$ & 0.631 & 1.074 & 6.96 & $1 Y, \mathrm{r}$ & 0.428 & 1.047 & 27.69 \\
\hline $2 X, \mathrm{f}$ & 0.164 & 1.019 & 4.41 & $2 Y, \mathrm{f}$ & 0.053 & 1.043 & 1.49 \\
\hline $3 X, \mathrm{r}$ & 0.370 & 0.996 & 12.92 & $3 Y, \mathrm{f}$ & 0.572 & 1.464 & 28.78 \\
\hline
\end{tabular}

Procedimiento Simplificado de Diseño PSD. En las Tablas 13 y 14 se anotan los cálculos para el piso 5 y en las 17 y 18 los resultados obtenidos a partir de los cortantes directos con la aplicación del PSD. Particularmente, en la Tabla 14 se anotan los cálculos de las fuerzas de diseño 
de algunos de los elementos estructurales, obtenidos a partir de los efectos producidos por los cortantes directos calculados en el paso 2 del método, corregidos por los FAT correspondientes.

Tabla 14. Fuerzas de diseño (en $t$ y $t-m$ ) en los elementos resistentes del edificio de cinco pisos calculados con el PSD. Sismo en dirección $X$.

\begin{tabular}{|c|c|c|c|c|c|c|c|}
\hline \multirow{2}{*}{$\begin{array}{c}\text { Col. en } \\
\text { ejes }\end{array}$} & \multicolumn{3}{|c|}{ Efectos del cortante directo } & \multirow[t]{2}{*}{$F A T$} & \multicolumn{3}{|c|}{ PSD } \\
\hline & $V$ & $M$ inf & M sup & & 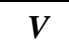 & $M$ inf & M sup \\
\hline $1 \mathrm{X}, 1 \mathrm{Y}$ & 2.15 & 3.21 & 3.25 & \multirow{3}{*}{1.074} & 2.31 & 3.45 & 3.49 \\
\hline $1 \mathrm{X}, 2 \mathrm{Y}$ & 2.23 & 3.34 & 3.36 & & 2.40 & 3.59 & 3.61 \\
\hline $1 X, 3 Y$ & 2.17 & 3.26 & 3.25 & & 2.33 & 3.50 & 3.49 \\
\hline $2 X, 1 Y$ & 1.45 & 2.16 & 2.18 & \multirow{3}{*}{1.019} & 1.48 & 2.20 & 2.22 \\
\hline $2 X, 2 Y$ & 1.49 & 2.23 & 2.24 & & 1.52 & 2.27 & 2.28 \\
\hline $2 X, 3 Y$ & 1.48 & 2.22 & 2.20 & & 1.51 & 2.26 & 2.24 \\
\hline $3 X, 1 Y$ & 4.15 & 6.20 & 6.23 & \multirow{3}{*}{0.996} & 4.13 & 6.18 & 6.21 \\
\hline $3 \mathrm{X}, 2 \mathrm{Y}$ & 4.40 & 6.58 & 6.61 & & 4.38 & 6.55 & 6.58 \\
\hline $3 X, 3 Y$ & 4.25 & 6.44 & 6.33 & & 4.23 & 6.41 & 6.30 \\
\hline
\end{tabular}

\section{DISCUSIÓN DE RESULTADOS}

Se discuten los resultados obtenidos en cada uno de los elementos resistentes de los entrepisos de las estructuras analizadas con cada uno de los métodos estudiados. Tomando en cuenta estos resultados se señalan las ventajas y desventajas de su aplicación desde el punto de vista de su utilización práctica.

En la Tabla 15 se presentan los cortantes totales en los dos elementos resistentes del edificio de un piso y en la Tabla 16 se anotan los errores relativos de estos cortantes, con respecto al método de Rosenblueth y Esteva (\%), calculados con diferentes criterios.

Tabla 15. Cortantes totales $(t)$ en los elementos resistentes del edificio de un piso calculados con diferentes métodos ( $\mathrm{f}=$ elemento flexible, $\mathrm{r}=$ =elemento rígido).

\begin{tabular}{|c|c|c|c|c|c|}
\hline Entrepiso & $\begin{array}{c}\text { Elemento } \\
\text { Tipo }\end{array}$ & \multicolumn{4}{|c|}{ Método } \\
\cline { 3 - 6 } & $\begin{array}{c}\text { Rosen- } \\
\text { blueth- } \\
\text { Esteva }\end{array}$ & Ávila & $\begin{array}{c}\text { Goel- } \\
\text { Chopra }\end{array}$ & PSD \\
\hline 1 & $1, \mathrm{f}$ & 559.10 & 554.48 & 554.57 & 559.12 \\
\cline { 2 - 6 } & $2, \mathrm{r}$ & 447.27 & 448.95 & 448.96 & 447.27 \\
\hline
\end{tabular}

Tabla 16. Errores relativos (\%) en cortantes totales en los elementos resistentes del edificio de un piso $(\mathrm{f}=$ elemento flexible, $\mathrm{r}=$ elemento rígido).

\begin{tabular}{|c|c|c|c|c|}
\hline \multirow{2}{*}{ Entrepiso } & $\begin{array}{c}\text { Elemento } \\
\text { Tipo }\end{array}$ & \multicolumn{3}{|c|}{ Método } \\
\cline { 3 - 5 } & & Ávila & $\begin{array}{c}\text { Goel- } \\
\text { Chopra }\end{array}$ & PSD \\
\hline \multirow{2}{*}{1} & $1, \mathrm{f}$ & -0.8 & -0.8 & 0.0 \\
\cline { 2 - 5 } & $2, \mathrm{r}$ & 0.4 & 0.4 & 0.0 \\
\hline
\end{tabular}


En la Tabla 17 se anotan los cortantes totales de los elementos resistentes del edificio de 5 pisos estudiado calculados con diferentes criterios y en la Tabla 18 se presentan fuerzas de diseño de elementos estructurales del mismo.

Tabla 17. Cortantes totales ( $\mathrm{t}$ ) en los elementos resistentes del edificio de cinco pisos calculados con diferentes métodos ( $\mathrm{f}=$ elemento flexible, $\mathrm{r}=$ elemento rígido).

\begin{tabular}{|c|c|c|c|c|c|c|c|c|c|c|}
\hline \multirow[t]{2}{*}{ Entrepiso } & \multirow{2}{*}{$\begin{array}{c}\text { Elemento } \\
\text { Tipo }\end{array}$} & \multicolumn{4}{|c|}{ Sismo en dirección $X$} & \multirow{2}{*}{$\begin{array}{c}\text { Elemento } \\
\text { tipo }\end{array}$} & \multicolumn{4}{|c|}{ Sismo en dirección $Y$} \\
\hline & & $\begin{array}{l}\text { Rosen- } \\
\text { blueth- } \\
\text { Esteva }\end{array}$ & Ávila & $\begin{array}{c}\text { Goel- } \\
\text { Chopra }\end{array}$ & PSD & & \begin{tabular}{|l|} 
Rosen- \\
blueth- \\
Esteva
\end{tabular} & Ávila & \begin{tabular}{|c|} 
Goel- \\
Chopra
\end{tabular} & PSD \\
\hline \multirow{3}{*}{5} & $1 X, \mathrm{f}$ & 6.97 & 6.66 & 6.71 & 6.96 & $1 Y, \mathrm{r}$ & 27.69 & 26.67 & 25.49 & 27.69 \\
\hline & $2 X, f$ & 4.41 & 4.36 & 4.34 & 4.41 & $2 Y, f$ & 1.49 & 1.47 & 1.49 & 1.49 \\
\hline & $3 X, r$ & 12.91 & 12.96 & 12.95 & 12.91 & $3 Y, f$ & 28.78 & 22.98 & 22.32 & 28.78 \\
\hline \multirow{4}{*}{4} & $1 X, \mathrm{f}$ & 14.05 & 13.76 & 13.78 & 14.05 & $1 Y, \mathrm{f}$ & 67.83 & 56.04 & 59.06 & 67.82 \\
\hline & $2 X, \mathrm{f}$ & 9.16 & 9.08 & 9.08 & 9.16 & $2 Y, f$ & 3.13 & 2.96 & 2.98 & 3.13 \\
\hline & $3 X, \mathrm{r}$ & 9.00 & 8.96 & 9.01 & 9.00 & $3 Y, r$ & 3.03 & 2.84 & 2.96 & 3.03 \\
\hline & $4 X, r$ & 17.96 & 17.96 & 17.96 & 18.01 & $4 Y, r$ & 46.43 & 40.16 & 43.16 & 46.44 \\
\hline \multirow{4}{*}{3} & $1 X, \mathrm{f}$ & 22.74 & 22.16 & 22.34 & 22.74 & $1 Y, f$ & 96.85 & 82.92 & 88.50 & 96.94 \\
\hline & $2 X, f$ & 13.32 & 13.12 & 13.22 & 13.32 & $2 Y, f$ & 3.97 & 3.8 & 3.88 & 3.97 \\
\hline & $3 X, \mathrm{r}$ & 13.12 & 13.08 & 13.11 & 13.12 & $3 Y, r$ & 4.10 & 3.88 & 3.92 & 4.10 \\
\hline & $4 X, \mathrm{r}$ & 26.30 & 26.08 & 26.24 & 26.30 & $4 Y, r$ & 72.68 & 60.68 & 66.20 & 72.63 \\
\hline \multirow{4}{*}{2} & $1 X, \mathrm{f}$ & 27.97 & 27.32 & 27.44 & 27.97 & $1 Y, f$ & 117.51 & 103.0 & 108.34 & 117.64 \\
\hline & $2 X, f$ & 16.41 & 16.24 & 16.26 & 16.41 & $2 Y, f$ & 4.87 & 4.72 & 4.76 & 4.87 \\
\hline & $3 X, r$ & 16.19 & 16.12 & 16.16 & 16.19 & $3 Y, r$ & 5.10 & 4.72 & 4.88 & 5.09 \\
\hline & $4 X, \mathrm{r}$ & 32.49 & 32.24 & 32.40 & 32.49 & $4 Y, r$ & 90.92 & 77.20 & 82.40 & 90.83 \\
\hline \multirow{4}{*}{1} & $1 X, \mathrm{f}$ & 31.49 & 30.80 & 31.06 & 31.49 & $1 Y, \mathrm{f}$ & 132.92 & 115.12 & 123.94 & 133.01 \\
\hline & $2 X, f$ & 18.52 & 18.36 & 18.42 & 18.52 & $2 Y, f$ & 5.50 & 5.28 & 5.40 & 5.5 \\
\hline & $3 X, r$ & 18.31 & 18.24 & 18.28 & 18.31 & $3 Y, r$ & 5.75 & 5.32 & 5.52 & 5.75 \\
\hline & $4 X, r$ & 36.79 & 36.40 & 36.64 & 36.78 & $4 Y, r$ & 102.61 & 85.76 & 94.40 & 102.55 \\
\hline
\end{tabular}

En la Fig. 4 se anotan los errores relativos, respecto al método de Rosenblueth-Esteva, de los cortantes totales de los elementos resistentes del edificio de cinco pisos calculados con diferentes métodos para la dirección $X$ y $Y$. En esta figura se aprecia que el PSD propuesto, proporciona prácticamente los mismos resultados que el método de Rosenblueth-Esteva, en los dos ejemplos analizados. Con el PSD, los valores del error relativo de los resultados no exceden el $0.1 \%$ que puede atribuirse a las operaciones aritméticas que intervienen en los cálculos.

Por su parte, los métodos de Ávila y de Goel-Chopra producen errores relativos de hasta $-20 \%$ en el elemento $3 Y$ del entrepiso 5 y superiores al $15 \%$ en el elemento $4 Y$ para los demás entrepisos en la dirección $Y$ del edificio. Mientras que en la dirección $X$ los valores del error relativo no exceden el 2.5\% para estos dos métodos, a excepción del elemento $1 X$ del entrepiso 5 , donde el valor del error relativo está alrededor del $4 \%$. En general se puede observar que, para el edificio de 5 pisos estudiado, los elementos de los bordes de las losas son los que producen los mayores valores de error relativo al calcular sus cortantes totales utilizando los métodos de Ávila y de Goel-Chopra. 

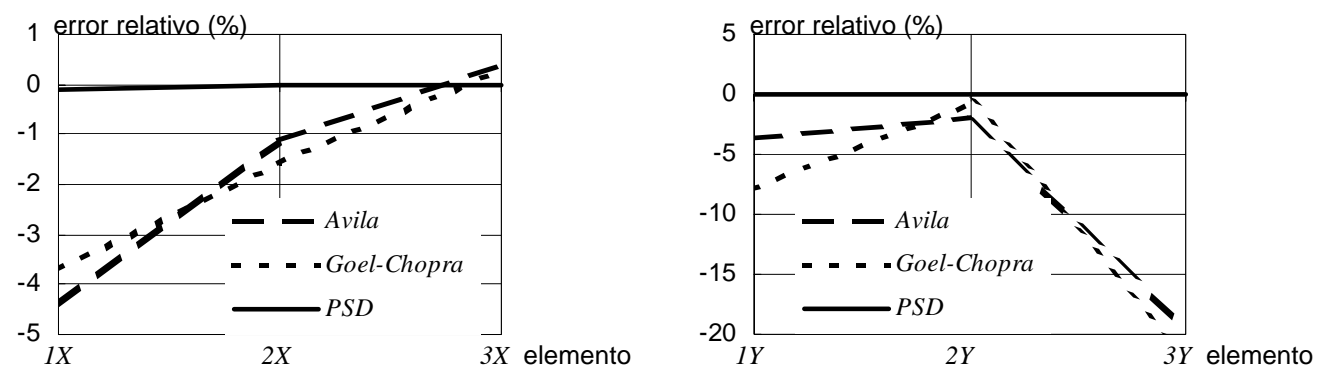

a) entrepiso 5
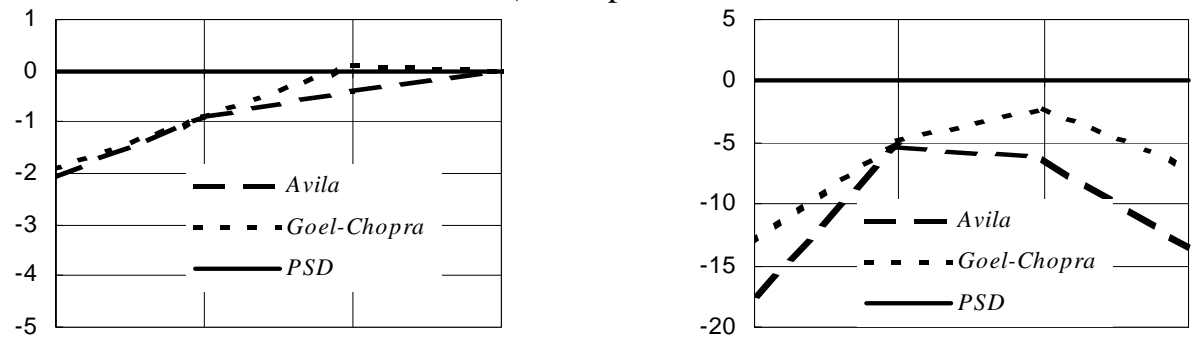

b) entrepiso 4

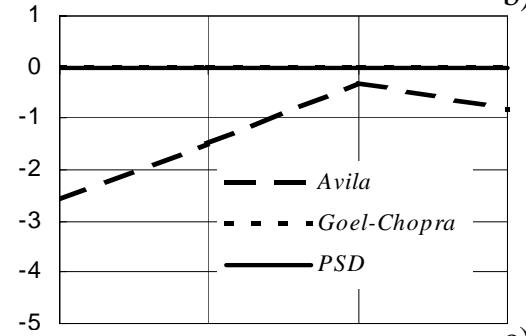

c) entrepiso $3^{-20}$
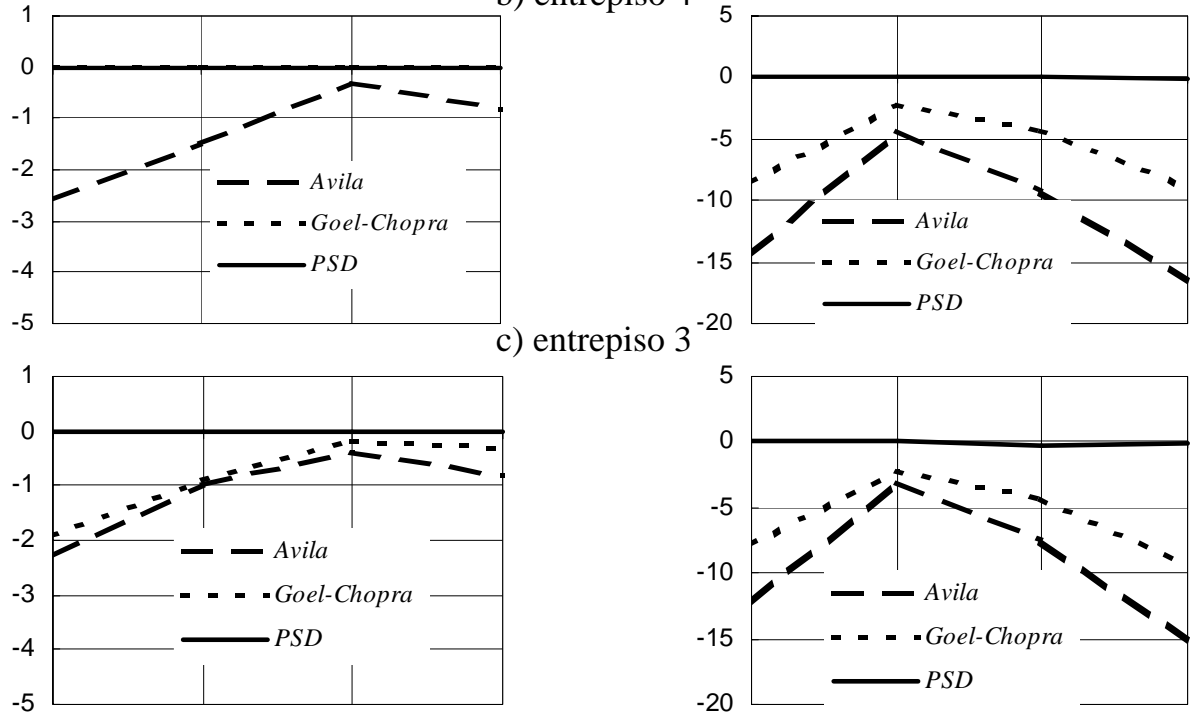

d) entrepiso $2^{-20}$
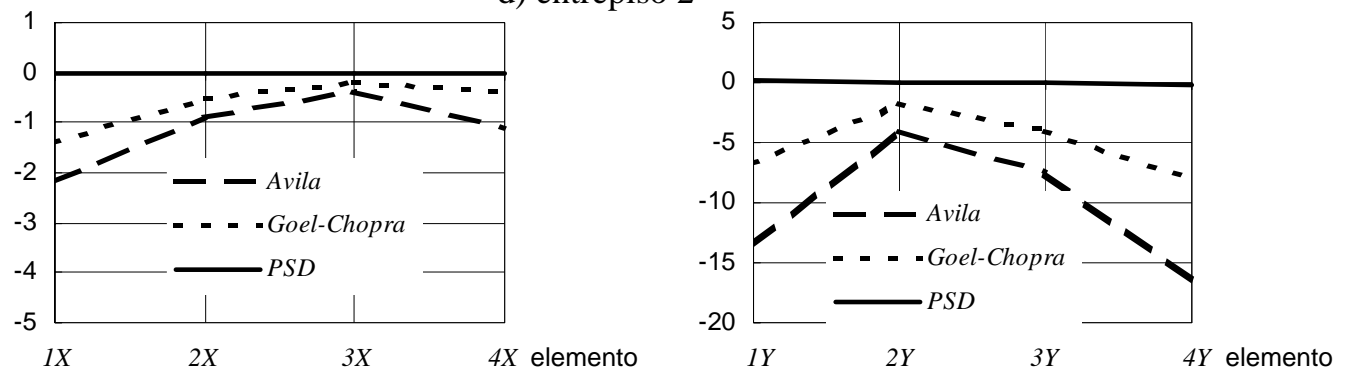

e) entrepiso 1

Figura 4. Errores relativos, respecto al método de Rosenblueth-Esteva, en cortantes totales de los elementos resistentes en dirección $X$ y $Y$ del edificio de cinco pisos calculados con diferentes métodos. 
Evaluación de Criterios de Diseño por Torsión Sísmica Estática para Edificos de Varios Pisos

Tabla 18. Fuerzas de diseño (en $t$ y $t-m$ ) en columnas del edificio de cinco pisos, calculadas con diferentes métodos de diseño por torsión sísmica estática. Edificio de cinco pisos.

\begin{tabular}{|c|c|c|c|c|c|c|c|c|}
\hline \multirow{2}{*}{ Entrepiso } & \multirow{2}{*}{$\begin{array}{c}\text { Columna } \\
\text { en ejes }\end{array}$} & \multicolumn{3}{|c|}{ Sismo en dirección $X$} & \multicolumn{3}{|c|}{ Sismo en dirección $Y$} & \multirow{2}{*}{ Método } \\
\hline & & $V$ & $M$ inf & M sup & $\boldsymbol{V}$ & M inf & M sup & \\
\hline \multirow{4}{*}{5} & \multirow{4}{*}{$2 X, 3 Y$} & 1.47 & 2.21 & 2.20 & 9.59 & 14.45 & 14.32 & Rosenblueth-Esteva \\
\hline & & 1.45 & 2.18 & 2.18 & 7.62 & 11.46 & 11.39 & Ávila \\
\hline & & 1.44 & 2.17 & 2.17 & 6.85 & 10.33 & 10.22 & Goel-Chopra \\
\hline & & 1.46 & 2.20 & 2.20 & 9.59 & 14.44 & 14.36 & $P S D$ \\
\hline \multirow{4}{*}{3} & \multirow{4}{*}{$2 X, 3 Y$} & 3.33 & 5.0 & 4.99 & 1.03 & 1.54 & 1.53 & Rosenblueth-Esteva \\
\hline & & 3.30 & 4.95 & 4.94 & 0.95 & 1.43 & 1.41 & Ávila \\
\hline & & 3.30 & 4.95 & 4.95 & 0.97 & 1.46 & 1.44 & Goel-Chopra \\
\hline & & 3.33 & 5.0 & 4.99 & 1.03 & 1.55 & 1.53 & PSD \\
\hline \multirow{4}{*}{1} & \multirow{4}{*}{$1 X, 1 Y$} & 7.87 & 15.71 & 15.78 & 33.23 & 69.40 & 63.50 & Rosenblueth-Esteva \\
\hline & & 7.70 & 15.43 & 15.38 & 28.78 & 58.70 & 56.41 & Ávila \\
\hline & & 7.74 & 15.51 & 15.43 & 31.86 & 65.43 & 61.99 & Goel-Chopra \\
\hline & & 7.87 & 15.71 & 15.77 & 33.23 & 69.80 & 64.10 & $P S D$ \\
\hline
\end{tabular}

Es importante mencionar que para decidir cuál método de los aquí evaluados presenta las mayores ventajas en su utilización práctica, deberán tomarse en cuenta, además de su precisión, factores como la cantidad de cálculos que requieren y/o la complejidad de su aplicación. Por ejemplo, los métodos de Ávila, Goel-Chopra y PSD permiten conocer directamente las fuerzas de diseño en los elementos resistentes (momentos flexionantes, fuerzas cortantes, axiales, etc.). A diferencia del método de Rosenblueth-Esteva en el que después de distribuir los cortantes directos y por torsión entre los elementos resistentes de cada entrepiso, es necesario hacer un análisis plano para cada elemento resistente aplicando las fuerzas totales calculadas.

Así, en la Tabla 19 se anotan las características básicas de los métodos estudiados. En ella se puede observar que en la aplicación del método de Ávila, inicialmente se necesita hacer dos modelos y dos análisis de la estructura, uno en cada una de sus direcciones ortogonales, para calcular los cortantes directos necesarios para determinar la excentricidad estructural. Para conocer las fuerzas de diseño en los elementos estructurales se deben hacer cuatro modelos y sus análisis correspondientes, uno para cada una de las nuevas coordenadas de los $C M$ de los entrepisos. Finalmente, los resultados provenientes de los dos análisis correspondientes a cada una de las direcciones ortogonales, se deben comparar para cada elemento estructural y para cada fuerza de diseño considerada y así elegir la más desfavorable. Esto es, para las trabes se considerarán al menos la fuerza cortante y el momento flexionante, mientras que para las columnas se tendrían que considerar al menos la fuerza axial, y los cortantes y momentos flexionantes biaxiales. Estos efectos corresponden al coeficiente que afecta al número de trabes y columnas indicado en la cuarta columna de la Tabla 19.

Con el método de Goel-Chopra, para calcular las fuerzas de diseño actuantes en los elementos estructurales se necesita hacer cuatro modelos y seis análisis de la estructura (tres en cada una de las direcciones ortogonales). Los resultados provenientes de estos análisis se deberán combinar de dos maneras diferentes. Al igual que con el método de Ávila, se deberá comparar 
cada elemento estructural para cada fuerza de diseño considerada y así elegir la más desfavorable (Tabla 19).

Tabla 19. Características básicas de los métodos estudiados.

\begin{tabular}{|c|c|c|c|}
\hline Método & $\begin{array}{c}\text { Núm. de } \\
\text { modelos }\end{array}$ & $\begin{array}{c}\text { Núm. de } \\
\text { análisis }\end{array}$ & $\begin{array}{c}\text { Comparaciones } \\
\text { para diseño }\end{array}$ \\
\hline Rosenblueth-Esteva & Ner & ner & 0 \\
Ávila & 6 & 6 & $2(2 \mathrm{nt}+5 \mathrm{nc})$ \\
Goel-Chopra & 4 & 6 & $4(2 \mathrm{nt}+4 \mathrm{nc})$ \\
PSD & 2 & 2 & 0 \\
\hline
\end{tabular}

Por su parte, el PSD requiere sólo de dos análisis, uno en cada una de las direcciones ortogonales del edifico para conocer las fuerzas debido al cortante directo en sus elementos estructurales. Las fuerzas de diseño se calculan al multiplicar estas fuerzas, producidas por los cortantes directos en los elementos estructurales, por los factores de amplificación por torsión FAT correspondientes. Los cortantes directos obtenidos de estos análisis son los mismos que se utilizan para calcular la excentricidad estructural. En este caso no es necesario hacer ninguna comparación para seleccionar las fuerzas de diseño en los elementos estructurales.

\section{CONCLUSIONES}

Se evaluaron diferentes propuestas para reducir la cantidad de cálculos en el análisis por computadora de modelos tridimensionales de edificios por torsión sísmica estática. Se describieron las bases teóricas que dan origen al análisis por torsión sísmica estática de estructuras de edificios; se estudiaron varias propuestas enfocadas a realizar este análisis mediante el uso de programas de análisis estructural comerciales. También se analizaron las dos definiciones de excentricidad estática que existen actualmente y se demostró que ambas proporcionan los mismos valores de momentos de torsión en los edificios de varios pisos.

Se propuso y evaluó un nuevo método denominado Procedimiento Simplificado de Diseño, $P S D$, el cual, sin perder precisión, reduce el número de análisis por lo menos a una tercera parte, con respecto a uno de los métodos más rápidos (método de Ávila). Se presentaron dos ejemplos en los que se aplican las diferentes propuestas. A partir de los resultados de los ejemplos estudiados se obtienen las siguientes conclusiones:

Para su aplicación, los métodos de Rosenblueth-Esteva y el de Ávila, requieren tomar en cuenta el signo de la excentricidad estructural. Con la clasificación de elementos rígidos y flexibles, necesaria para aplicar el $P S D$, este aspecto se evita, ya que en él se utiliza el valor absoluto de este parámetro. Además de que con esta clasificación es posible tener una mejor interpretación de la distribución de las fuerzas entre los elementos resistentes al utilizar una ecuación correspondiente a cada tipo de ellos.

El método de Ávila, al igual que el de Goel-Chopra y el PSD permiten conocer directamente las fuerzas para diseño en los elementos resistentes. 
Debido a que se utilizan las mismas ecuaciones, con el PSD propuesto se obtuvieron prácticamente los mismos resultados que con el método de Rosenblueth-Esteva en los ejemplos analizados.

Para calcular las fuerzas de diseño actuantes en los elementos estructurales con el método de Rosenblueth-Esteva, después de distribuir los cortantes directos y por torsión entre los elementos resistentes de cada entrepiso, es necesario hacer un análisis individual en el plano de cada uno de ellos utilizando las fuerzas cortantes totales calculadas con estos.

La aplicación del método de Ávila requiere de seis modelos diferentes de la estructura que se analiza; dos para calcular los cortantes directos y con ellos la excentricidad estática, y uno para cada una de las nuevas posiciones de $C M$ en cada una de las direcciones ortogonales para obtener las fuerzas de diseño en los elementos estructurales.

Por otro lado, a pesar de que con el método de Goel-Chopra no es necesario calcular la posición de $C T$, su aplicación requiere de cuatro modelos estructurales, dos con giros impedidos alrededor del eje vertical de las losas, y dos con estos grados de libertad permitidos. Para calcular las fuerzas de diseño se necesita hacer dos análisis de la estructura en cada dirección ortogonal, uno con los giros de las losas libres y otro con estos grados de libertad fijos, este último con dos condiciones de carga.

Para conocer las fuerzas para diseño en los elementos resistentes con los métodos de Ávila y de Goel-Chopra, es necesario comparar, elemento a elemento, los resultados de dos análisis y de cuatro combinaciones de carga, respectivamente.

Finalmente, el PSD requiere únicamente de dos análisis de la estructura, uno en cada una de sus direcciones ortogonales. Estos análisis son los mismos con los que se obtienen los cortantes directos necesarios para calcular la excentricidad estructural. Las fuerzas de diseño en los elementos estructurales se obtienen corrigiendo, con los factores de amplificación por torsión FAT correspondientes, los elementos mecánicos producidos por el cortante directo en los elementos estructurales.

\section{REFERENCIAS}

Ávila, J A (1991), “Comentarios y Ejemplos a las Normas Técnicas Complementarias para Diseño por Sismo, DDF”, Series del Instituto de Ingeniería, ES-7, UNAM, México.

Bazán, E (1978), “Análisis sísmico de edificios con muros rigidizantes”, IMCYC, Vol. 41, No. 91, México D.F., México.

Bazán, E y R Meli (2000), Diseño sísmico de edificios, Editorial Limusa, México.

Cheung, V. W-T y W K Tso (1986), “Eccentricity in irregular multistorey buildings”, Canadian Journal of Civil Engineering, Vol. 13, No. 1, pp. 46-52.

Damy, J (1988), “Comentarios al inciso 8.6 de las Normas Técnicas Complementarias para Diseño por Sismo”, Revista de Ingeniería Sísmica, SMIS, No. 33, pp. 66-99. 
Escobar, J A, J A Mendoza y R Gómez (2002), "Procedimiento simplificado para diseño por torsión sísmica estática”, Memorias, XIII Congreso Nacional de Ingeniería Estructural, Puebla, Puebla, México, CD-ROM.

Escobar, J A, J A Mendoza y R Gómez (2004), “Diseño simplificado por torsión sísmica estática”, Revista de Ingeniería Sísmica, SMIS, No. 70, enero-junio, pp. 77-107.

Goel, R K y A K Chopra (1992), "Seismic code analysis of buildings without locating centers of rigidity”, ASCE Journal of Structural Engineering, Vol. 119, No. 10, 3039-3055.

NTC-95 (1995), “Normas Técnicas Complementarias para Diseño por Sismo”, Gaceta Oficial del Departamento del Distrito Federal., 7 de noviembre de 1994.

RCDF-95 (1995), “Reglamento de Construcciones para el Distrito Federal”, México.

Rosenblueth, E (1979), “Seismic design requirements in a 1976 Mexican Code”, Earthquake Engineering and Structural Dynamics, Vol. 7, pp. 49-61.

Rosenblueth, E. y L Esteva (1962), Diseño sísmico de edificios, Folleto Complementario al Reglamento de Construcciones para del Distrito Federal, México D.F., México.

Tso, W K (1990), "Static eccentricity concept for torsional moment estimations", ASCE Journal of Structural Engineering, Vol. 16, No. 5, pp. 1199-1212.

\section{AGRADECIMIENTOS}

El primer autor agradece a J.A. Ávila, S. Loera y D. Murià Vila sus valiosos comentarios y la revisión crítica del presente escrito. La etapa inicial de este estudio fue patrocinada por la Dirección General de Obras del Gobierno del Distrito Federal.

\section{APÉNDICE A. CÁlCULOS PARA LA ESTRUCTURA DE UN PISO}

Centro de torsión

$$
x_{C T}=\frac{12000(12)}{15000}=9.6 \mathrm{~m}
$$

Excentricidad estática

$$
e_{s}=9.6-6.0=3.6 \mathrm{~m}
$$

Excentricidad de diseño, ecuaciones (14)

$$
\begin{gathered}
e d_{1}=1.5(3.6)+0.1(12)=6.6 m \\
e d_{2}=3.6-0.1(12)=2.4 m
\end{gathered}
$$


Momentos de torsión

$$
\begin{aligned}
& M_{11}=745.46(6.6)=4920.04 t-m \\
& M_{12}=745.46(2.4)=1789.10 t-m
\end{aligned}
$$

\section{Método de Rosenblueth-Esteva}

Cortantes directos, ecuación (2)

$$
\begin{gathered}
V d_{1}=\frac{745.46}{15000}(3000)=149.1 t \\
V d_{2}=\frac{745.466}{15000}(12000)=596.37 t
\end{gathered}
$$

Cortantes por torsión, ecuación (15)

$$
\begin{aligned}
& V t_{1}=V t_{2}=\frac{4920.04}{345600}(28800)=410.0 t \\
& V t_{1}=V t_{2}=\frac{1789.10}{345600}(28800)=149.1 t
\end{aligned}
$$

\section{Método de Ávila}

Excentricidad estructural, ecuación (6)

$$
e_{s}=6.0-9.6=-3.6 m
$$

Excentricidad de diseño, ecuaciones (20)

$$
\begin{gathered}
E d_{1}=1.5(-3.6)+0.1(-12)=-6.6 \mathrm{~m} \\
E d_{2}=-3.6-0.1(-12)=-2.4 \mathrm{~m}
\end{gathered}
$$

Nuevas coordenadas del $C M$, ecuación (18)

$$
\begin{aligned}
& x{ }_{C M 1}=9.6-6.6=3.0 \mathrm{~m} \\
& x^{*}{ }_{C M 2}=9.6-2.4=7.2 \mathrm{~m}
\end{aligned}
$$




\section{Método de Goel-Chopra}

Al hacer las combinaciones de carga especificadas en el método, para el elemento 1 se obtiene

$$
\begin{gathered}
V t 1=-0.5(148.92)+1.5(370.21)+73.71=554.57 t \\
V t 2=370.21-73.71=296.50 t
\end{gathered}
$$

y para el elemento 2

$$
\begin{gathered}
V t 1=-0.5(596.54)+1.5(375.25)-73.71=190.01 t \\
V t 2=375.25+73.71=448.96 t
\end{gathered}
$$

\section{Procedimiento Simplificado de Diseño, PSD}

Radio de giro del entrepiso, ecuación (28)

$$
\rho=\frac{1}{12} \sqrt{\frac{149.1(9.6)^{2}+596.37(2.4)^{2}}{745.46}}=0.4 \Rightarrow \rho^{2}=0.16
$$

Excentricidad estática normalizada

$$
e=|3.6 / 12|=0.3
$$

A partir de su ubicación (Fig. 3), el elemento 1 se clasifica como elemento flexible, por lo tanto, de las ecuaciones (27) se tiene que

$$
\zeta_{1}=\frac{9.6}{12}=0.8 ; \mathrm{FATf}_{1}=1+\frac{0.8}{0.16}[0.1+1.5(0.3)]=3.75
$$

El elemento 2 es un elemento rígido según su ubicación, entonces

$$
\zeta_{2}=\frac{2.4}{12}=0.2 ; \text { FATr }_{2}=1+\frac{0.2}{0.16}[0.1-0.3]=0.75
$$




\section{APÉNDICE B. CÁLCULOS PARA EL ENTREPISO CINCO DEL EDIFICIO DE CINCO PISOS}

Tabla B1. Ubicación en m, de CM, CC, CT, y CR (Escobar et al., 2004)

\begin{tabular}{|c|c|c|c|c|c|c|c|c|}
\hline $\begin{array}{c}\text { Piso } \\
\text { Entrepiso }\end{array}$ & $x_{C M}$ & $y_{C M}$ & $x_{C C}$ & $y_{C C}$ & $x_{C T}$ & $y_{C T}$ & $x_{C R}$ & $y_{C R}$ \\
\hline 5 & 6.75 & 3.75 & & & 5.78 & 4.73 & & \\
\hline 5 & & & 6.75 & 3.75 & & & 5.78 & 4.73 \\
\hline
\end{tabular}

\section{Método de Rosenblueth-Esteva}

Sismo en dirección $X$

$$
\begin{gathered}
V d_{1 X}=\frac{23.77(1200)}{4400}=6.48 t ; V d_{2 X}=\frac{23.77(800)}{4400}=4.32 t ; V d_{3 X}=\frac{23.77(2400)}{4400}=12.97 t \\
e_{s}=4.73-3.75=0.98 \mathrm{~m} ; \quad e d_{1}=1.5(0.98)+0.1(7.5)=2.22 \mathrm{~m} ; \quad e d_{2}=0.98-0.1(7.5)=0.23 \mathrm{~m} \\
M_{51}=23.77(2.22)=52.77 \mathrm{t}-\mathrm{m} ; \quad M_{52}=23.77(0.23)=5.47 \mathrm{t}-\mathrm{m}
\end{gathered}
$$

De las Tablas 7 y 8 :

$$
\begin{gathered}
K_{\theta}=46472.76+575160.72=621633.48 t-\mathrm{m}^{2} \\
V t 1_{1 X}=\frac{5672.73(52.77)}{621633.48}=0.48 t ; \quad V t 2_{1 X}=\frac{5672.73(5.47)}{621633.48}=0.05 t \\
V t 1_{2 X}=\frac{981.82(52.77)}{621633.48}=0.08 t ; \quad V t 2_{2 X}=\frac{981.82(5.47)}{621633.48}=0.01 t \\
V t 1_{3 X}=\frac{6654.55(52.77)}{621633.48}=0.56 t ; \quad V t 2_{3 X}=\frac{6654.55(5.47)}{621633.48}=0.06 t
\end{gathered}
$$

Sismo en dirección $Y$

$$
\begin{gathered}
V d_{1 X}=\frac{47.54(7400)}{13300}=26.45 t ; V d_{2 X}=\frac{47.54(400)}{13300}=1.43 t ; V d_{3 x}=\frac{47.54(5500)}{13300}=19.66 t \\
e_{s}=6.75-5.78=0.97 \mathrm{~m} ; \quad e d_{1}=1.5(0.97)+0.1(13.5)=2.81 \mathrm{~m} ; \quad e d_{2}=0.97-0.1(13.5)=-0.38 \mathrm{~m} \\
M_{51}=47.54(2.81)=133.58 t-m ; \quad M_{52}=47.54(-0.38)=-18.07 t-m \\
V t 1_{1 Y}=\frac{42772(133.58)}{621633.48}=9.19 t ; \quad V t 2_{1 Y}=\frac{42772(18.07)}{621633.48}=1.24 t
\end{gathered}
$$




$$
\begin{gathered}
V t 1_{2 Y}=\frac{288(133.58)}{621633.48}=0.06 t ; \quad V t 2_{2 Y}=\frac{288(18.07)}{621633.48}=0.01 t \\
V t 1_{3 Y}=\frac{42460(133.58)}{621633.48}=9.12 t ; \quad V t 2_{3 Y}=\frac{42460(18.07)}{621633.48}=1.23 t
\end{gathered}
$$

\section{Método de Ávila}

Sismo en dirección $X$

$$
\begin{gathered}
y_{C M}=3.75 \mathrm{~m} ; \quad y_{C T}=4.73 \mathrm{~m} ; \quad e_{s}=3.75-4.73=-0.98 \mathrm{~m} \\
E d_{1}=1.5(-0.98)+0.1(-7.5)=-2.22 \mathrm{~m} ; \quad E d_{2}=-0.98-0.1(-7.5)=-0.23 \mathrm{~m} \\
y^{*}{ }_{C M 1}=4.73-2.22=2.51 \mathrm{~m} ; \quad y^{*}{ }_{C M 2}=4.73-0.23=4.50 \mathrm{~m}
\end{gathered}
$$

Sismo en dirección $Y$

$$
\begin{gathered}
x_{C M}=6.75 \mathrm{~m} ; \quad x_{C T}=5.78 \mathrm{~m} ; \quad e_{s}=6.75-5.78=0.97 \mathrm{~m} \\
E d_{1}=1.5(0.97)+0.1(13.5)=2.81 \mathrm{~m} ; \quad E d_{2}=0.97-0.1(13.5)=-0.38 \mathrm{~m} \\
x^{*}{ }_{C M 1}=5.78+2.81=8.59 \mathrm{~m} ; \quad x^{*}{ }_{C M 2}=5.78-0.38=5.40 \mathrm{~m}
\end{gathered}
$$

\section{Procedimiento Simplificado de Diseño, PSD}

Sismo en dirección $X$

$$
y_{C C}=3.75 \mathrm{~m} ; \quad y_{C R}=4.73 \mathrm{~m} ; \quad e_{s}=3.75-4.73=-0.98 \mathrm{~m}
$$

$\sum V d y_{i} x_{i}^{2} / d y_{j}+\sum V d x_{i} y_{i}^{2} / d x_{j}=\left(6.48(4.73)^{2}+4.32(1.23)^{2}+12.97(2.77)^{2}\right) / 0.54+$

$$
\begin{array}{r}
+\left(26.45(5.78)^{2}+1.43(0.72)^{2}+19.66(7.72)^{2}\right) / 0.357=6223.45 t-m \\
\rho_{x 5}=\frac{1}{7.5} \sqrt{\frac{6223.45}{23.77 / 0.54}}=1.585 \Rightarrow \rho^{2}=2.511 ; \quad e=|-0.98 / 7.5|=0.13
\end{array}
$$

Cortante total para los elementos flexibles, $1 X$ y $2 X$

$$
\zeta_{1 X}=\frac{4.75}{7.5}=0.63 ; \text { FATf }_{1 X}=1+\frac{0.63}{2.511}(0.1+1.5(0.13))=1.074 ; V_{1 X}=1.074(6.48)=6.96 t
$$




$$
\zeta_{2 X}=\frac{1.23}{7.5}=0.164 ; \text { FATf }_{2 X}=1+\frac{0.164}{2.511}(0.1+1.5(0.13))=1.019 ; V_{2 X}=1.019(4.32)=4.41 t
$$

Para el elemento rígido $3 X$, se obtiene

$$
\zeta_{3 X}=\frac{2.78}{7.5}=0.37 ; \text { FATr }_{3 X}=1+\frac{0.369}{2.511}(0.1-0.13)=0.996 ; V_{3 X}=0.996(12.97)=12.92 t
$$

Sismo en dirección $Y$

$$
\begin{gathered}
x_{C C}=6.75 m ; x_{C R}=5.78 m ; e_{s}=6.75-5.78=0.97 m \\
\rho_{y 5}=\frac{1}{13.5} \sqrt{\frac{6223.45}{47.54 / 0.358}}=0.506 \Rightarrow \rho^{2}=0.256 ; e=|0.97 / 13.5|=0.0719
\end{gathered}
$$

Para los dos elementos flexibles $2 Y$ y $3 Y$, se tiene que

$$
\begin{gathered}
\zeta_{2 Y}=\frac{0.72}{13.5}=0.053 ; \text { FATf }_{2 Y}=1+\frac{0.053}{0.256}(0.1+1.5(0.0719))=1.043 ; V_{2 Y}=1.043(1.43)=1.49 t \\
\zeta_{3 Y}=\frac{7.72}{13.5}=0.572 ; \text { FATf }_{3 Y}=1+\frac{0.572}{0.256}(0.1+1.5(0.0719))=1.464 \\
V_{3 Y}=1.464(19.66)=28.78 t
\end{gathered}
$$

Para el elemento rígido $1 Y$ se obtiene

$$
\zeta_{1 Y}=\frac{5.78}{13.5}=0.428 ; \text { FATr }_{1 Y}=1+\frac{0.428}{0.256}(0.1-0.0719)=1.047 ; V_{1 Y}=1.047(26.45)=27.69 t
$$

